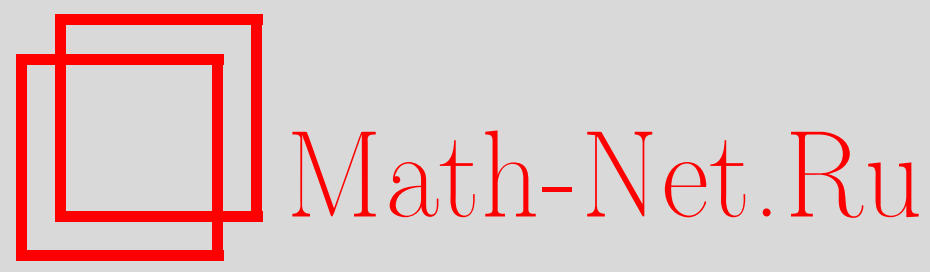

О. Н. Агеев, О спектре декартовых степеней классических автоморфизмов, Матем. заметки, 2000, том 68, выпуск 5, 643-647

DOI: https://doi.org/10.4213/mzm985

Использование Общероссийского математического портала Math-Net.Ru подразумевает, что вы прочитали и согласны с пользовательским соглашением http://www.mathnet.ru/rus/agreement

Параметры загрузки:

IP: 54.162 .27 .143

26 апреля 2023 г., $12: 42: 20$

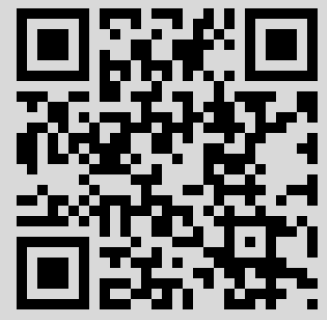




\section{О СПЕКТРЕ ДЕКАРТОВЫХ СТЕПЕНЕЙ КЛАССИЧЕСКИХ АВТОМОРФИЗМОВ}

\section{O.Н. Агеев}

В статье доказанаследующая теорема: для декартовых степеней $T^{(n)}$ преобразования Чекона $T$ функция кратности спектра принимает набор значений $\{n, n(n-1), \ldots$, $n !\}$ в подпространстве $\{\text { const }\}^{\perp}$ или, эквивалентно, оператор $T^{(n)}$ имеет простой спектр в подпространстве $C_{\text {sim }}$ всех симметричных функций относительно группы координатных перестановок. Непосредственньм следствием этой теоремы является попарная взаимная сингулярность всех сверток меры максимального спектрального типа преобразования $T$. При $n=2 T \times T$ имеет однородный двукратный спектр в $\{\text { const }\}^{\perp}$, т.е. решение задачи Рохлина для преобразования Чекона. Обсуждается справедливость этой теоремы для других классических автоморфизмов.

Библиограффия: 8 названий.

1. Рассмотрим кусочно-линейные, сохраняюшие ориентацию и меру Лебега $l$ преобразования полуинтервала $[0,1)=I$. Преобразование $T$ и оператор в $L_{2}(l): T f(x)=$ $f(T x)$ часто назьвают автоморфизмами и обозначают одним символом $T$.

Для автоморфизма $T$ назовем башней набор дизъюнктных полуинтервалов $\left\{J_{i}\right\}_{i=1}^{q}$, где $\left|J_{i}\right|=\left|J_{j}\right|(i, j=1, q)$ и $T$ линейно отображает $J_{i}$ на $J_{i+1}(i=1, q-1)$. Множество $J_{i}$ назьвается $i$-м этажсом башни, $q$-ее высотой, $J_{q}$-ее верхом.

Будем использовать следующий вариант определения классического преобразования $T$ Чекона. При $n=1$ имеем башню $C_{1}=\{[0,2 / 3)\}$. На $n$-й стадии имеем башню $C_{n}=\left\{J_{1}, \ldots, J_{q_{n}}\right\}$ и $T$ не определен на $J_{q_{n}} \cup\left[1-1 / 3^{n}, 1\right)$, где $q_{n}=\left(3^{n}-1\right) / 2,\left|J_{k}\right|=2 / 3^{n}$ и $J_{1} \cup \cdots \cup J_{q_{n}}=\left[0,1-1 / 3^{n}\right)$. Разобьем $J_{k}$ на полуинтервалы $J_{k, 0}, J_{k, 1}, J_{k, 2}$ равной длины. Тогда подбашни $\left\{J_{1, i}, J_{2, i}, \ldots, J_{q_{n}, i}\right\}(i=0,1,2)$ получены разрезанием (cutting) $C_{n}$. Башня $C_{n+1}$ получена надставкой (stacking) $(i+1)$-й подбашни наверх $i$-й, со вставкой 1-го этажа между 1-й и 2-й. Поэтому

$$
C_{n+1}=\left\{J_{1,0}, \ldots, J_{q_{n}, 0}, J_{1,1}, \ldots, J_{q_{n}, 1}, J^{\prime}, J_{1,2}, \ldots, J_{q_{n}, 2}\right\}
$$

и $T$ не определен на $J_{q_{n}, 2} \cup\left[1-1 / 3^{n+1}, 1\right)$, где $J^{\prime}=\left[1-1 / 3^{n}, 1-1 / 3^{n+1}\right)$. Следовательно, на этом шаге мы определили $T$ на множестве $J_{q_{n}, 0} \cup J_{q_{n}, 1} \cup J^{\prime}$.

Выделим работы [1]-[3], изучающие это преобразование и его обобщения. Рассмотрим его спектральные свойства. Пусть $T$ есть унитарный оператор в $L_{2}(l)$. Мы используем следующие обозначения: символ “ $\rightarrow$ " для слабой операторной сходимости;

Работа выполнена при частичной финансовой поддержке программы "Ведущие научные школы”, грант № 96-15-96135, и Российского фонда фундаментальных исследований, грант № 01-01104. 
$C_{T}\left(f_{i}: i \in A\right)$ - наименьшее замкнутое подпространство в $L_{2}(l)$, содержащее векторы $T_{f_{i}}^{n}, n \in \mathbb{Z} ; T^{n}=T \times \cdots \times T(n$ раз $) ; \sigma_{T}(f)$ - единственная борелевская мера на $\mathbb{T}=\{\lambda \in \mathbb{C}:|\lambda|=1\}$ с $\widehat{\sigma}_{T}(f)(n)=\left(T^{n} f, f\right) ; \sigma_{T}$ - мера максимального спектрального типа (т.е. максимальная среди $\left.\sigma_{T}(f)\right) ; \nu(\cdot \mid c)$ - система канонических условных мер для $\sigma_{T}^{n}=\sigma_{T} \times \cdots \times \sigma_{T}$ на слоях $C=\left\{\left(\lambda_{1}, \ldots, \lambda_{n}\right): \lambda_{1} \cdots \lambda_{n}=c\right\} ; \sigma^{(n)}$ - образ меры $\sigma^{n}$ при отображении $\left(\lambda_{1}, \ldots, \lambda_{n}\right) \rightarrow \lambda_{1} \cdots \lambda_{n} ; M(T)$ - множество существенных спектральных кратностей оператора $T ; m(T)=\max \{k: k \in M(T)\}$.

Мы говорим, что $T$ имеет простой спектр, если $m(T)=1$. Тогда $L_{2}(l)=C_{T}(\varphi)$ для некоторого $\varphi(x)$. Обозначим $\varphi_{k}(x)=\varphi\left(x_{1}\right) \cdots \varphi\left(x_{k}\right), C_{\mathrm{sim}}(n)$ - пространство таких $f$ из $L_{2}\left(I^{n}, l^{n}\right)$, которые инвариантны относительно любых перестановок координат; $H^{\left\{i_{1}, \ldots, i_{k}\right\}} C_{E}\left(T_{\varphi_{0}}^{j_{1}}\left(x_{i_{1}}\right) \cdots T_{\varphi_{0}}^{j_{k}}\left(x_{i_{k}}\right): j_{m} \in \mathbb{Z}\right\}$, где $E$ - тождественный оператор, $\varphi_{0}(x)=\varphi(x)-\int \varphi(x) d l$. Хорошо известно, что для преобразования Чекона $T m(T)=1$ и $\sigma_{T}=\sigma_{T}(\varphi)=\sigma_{T}^{\prime}+\delta$, где $\sigma_{T}^{\prime}=\sigma_{T}\left(\varphi_{0}\right)$ непрерывна и $\operatorname{supp} \delta=\{1\}$.

В работе [4] доказано, что $\sigma_{T}^{\prime(n)} \perp \sigma_{T}^{\prime(m)}$ при $m \neq n$.

Теорема 1. Если $T$ - преобразование Чекона, то для любого $n$

$$
M\left(T^{n}\right)=\{n, n(n-1), \ldots, n !\} \quad \text { в } \quad\{\text { const }\}^{\perp} .
$$

СлЕДСТВИЕ 1. При $n=2$ имеем конкретный пример преобразования с однородным спектром кратности 2. (Это было впервые установлено в [5] и является решением задачи Рохлина.)

СлЕДСТВИЕ 2. Если $m \neq n$, mo $\sigma_{T}^{\prime(n)} \perp \sigma_{T}^{\prime(m)}$.

ДокАЗАТЕЛЬСТво. Пусть $n>m$. Ясно, что мера максимального спектрального типа оператора $T^{(n)}$ в $H^{\{1, \ldots, k\}}$ есть $\sigma_{T}^{\prime(k)}$. Из непрерьвности $\sigma_{T}^{\prime}$ имеем (см. [5])

$$
M\left(\left.T^{(n)}\right|_{H\{1, \ldots, k\}}\right)=M\left(\left.T^{(k)}\right|_{H}\{1, \ldots, k\}\right) \subseteq k ! \mathbb{N} .
$$

Если $\sigma_{T}^{\prime(m)} \not \supset \sigma_{T}^{\prime(n)}$, то существует мера $\nu$, являющаяся их общей компонентой. Поэтому пространства $H^{\{1, \ldots, m\}}$ и $H^{\{1, \ldots, n\}}$ ортогональны и имеют $m$ ! и $n$ ! попарно ортогональных подпространств $C_{T^{(n)}}\left(f_{i}\right)$, где $\sigma_{T^{(n)}}\left(f_{i}\right)=\nu$. Следовательно, $m\left(T^{(n)}\right) \geqslant$ $m !+n !$. Это противоречит теореме 1 .

ЗАмечАниЕ 1. Фактически, свойство (*) будет доказано ниже для всех операторов (не обязательно преобразований), удовлетворяющим условиям леммы 1 и $(* *)$, где $\theta_{k}$ принимает $n-1$ различных значений.

ЗАМЕчАнИЕ 2. Свойство (*) для типичного $T$ было установлено в [5], что есть в точности ответ на вопрос Катка [6]. Однако часто проще проверить некоторое свойство для элемента, чем доказать, что он из типичного множества с этим свойством.

Лемма 1. Если унитарный оператор Т имеет простой непрерывный спектр в инвариантном подпространстве $\{\text { const }\}^{\perp}$, то следующие свойства әквивалент$H b l$ :

1) onepamop $T^{(n)}$ uмеeт простой спектр в $C_{\text {sim }}(n)$;

2) $M\left(T^{(n)}\right)=\{n, n(n-1), \ldots, n !\}$ в $\{\text { const }\}^{\perp}$;

3) $\operatorname{supp} \nu_{n}(\cdot \mid c)$ состоит из одной точки с точностью до перестановки координат для п.в. с по мере $\sigma_{T}^{(n)}$;

4) $C_{\operatorname{sim}}(n)=C_{T^{(n)}}\left(\varphi_{n}\right)$. 
ДокАЗАТЕЛЬСТво. Удобно перейти к спектральному представлению оператоpa $T^{(n)}$. Оператор $T^{j_{1}} \times T^{j_{2}} \times \cdots \times T^{j_{n}}$ в $L_{2}\left(I^{n}, l^{n}\right)$ спектрально изоморфен оператору $\lambda^{j_{1}} \cdots \lambda^{j_{n}} \cdot E$ в $L_{2}\left(\mathbb{T}^{n}, \sigma_{T}^{n}\right)$. Функция спектральной кратности оператора $\tilde{T}^{(n)}=\lambda_{1} \cdots \lambda_{n} \cdot E$ в точке $c$ равна числу точек в носителе меры $\nu_{n}(\cdot \mid c)$. Функции $\varphi_{n}$ будет соответствовать $\mathbf{1}$, функции $\varphi_{k}(k<n)$ будет соответствовать функция с носителем в множестве $\left\{\left(\lambda_{1}, \ldots, \lambda_{n}\right): \lambda_{k+1}=\cdots=\lambda_{n}=1\right\}$. Аналогичное представление имеет $T^{(n)}$ и в $H^{\left\{i_{1}, \ldots, i_{k}\right\}}$, но вместо $\sigma_{T}^{n}$ будет $\sigma_{T}^{\prime k}$.

Ясно, что $C_{T^{(n)}}\left(\varphi_{n}\right) \subseteq C_{\mathrm{sim}}(n)$ и $\sigma_{T^{(n)}}\left(\varphi_{n}\right)=\sigma_{T}^{(n)}$. Поэтому 1$\left.) \Longleftrightarrow 4\right)$.

Из 3) следует, что любая симметрическая функция $\varphi_{\operatorname{sim}}\left(\lambda_{1}, \ldots, \lambda_{n}\right)$ равна функции, постоянной на каждом слое $C=\left\{\left(\lambda_{1}, \ldots, \lambda_{n}\right): \lambda_{1} \cdots \lambda_{n}=c\right\}$ для п.в. $\left(\lambda_{1}, \ldots, \lambda_{n}\right)$ по мере $\sigma_{T}^{n}$. Поэтому $\varphi_{\operatorname{sim}} \in C_{\tilde{T}(n)}(\mathbf{1})$ и это влечет 4$)$.

Пусть неверно 3). Тогда нетрудно построить две симметрические функции $f_{1}, f_{2} \mathrm{c}$ дизъюнктньми носителями, удовлетворяющие свойству

$$
\int_{C} f_{i} \cdot d \nu_{n}(\cdot \mid c)=\psi(c) \not \equiv 0 .
$$

Следовательно, $\sigma_{\tilde{T}(n)}\left(f_{1}\right)=\sigma_{\tilde{T}^{(n)}}\left(f_{2}\right)$. Учитьвая, что $C_{\tilde{T}^{(n)}}\left(f_{1}\right)$ и $C_{\tilde{T}^{(n)}}\left(f_{2}\right)$ ортогональны, получим, что 1) неверно.

Мера $\sigma_{T}^{n}$ имеет компоненту, эквивалентную $\sigma_{T}^{k} \times \delta^{n-k}$, где $\operatorname{supp} \delta=\{1\}$. Поэтому

$$
\operatorname{supp} \nu_{n}(\cdot \mid c) \cap\left\{\left(\lambda_{1}, \ldots, \lambda_{n}\right): \lambda_{k+1}=\cdots=\lambda_{n}=1\right\} \supseteq \operatorname{supp} \nu_{k}(\cdot \mid c) .
$$

Отсюда следует 3$)$ и для $\nu_{k}(\cdot \mid c)$, где $k=1, \ldots, n$. Поэтому

$$
M\left(\left.T^{(n)}\right|_{H^{\left\{i_{1}, \ldots, i_{k}\right\}}}\right)=M\left(\left.T^{(k)}\right|_{H\{1, \ldots, k\}}\right) \subseteq M\left(T^{(k)}\right) \subseteq\{1, \ldots, k\}
$$

для любого набора $1 \leqslant i_{1}<i_{2}<\cdots<i_{k} \leqslant n$. Учитьвая $(1)$, получим

$$
M\left(\left.T^{(n)}\right|_{\left.H^{\left\{i_{1}, \ldots, i_{k}\right.}\right\}}\right)=\{k !\} .
$$

Учитывая (2), как и при доказательстве следствия 2, получим $\sigma_{T}^{\prime(k)} \perp \sigma_{T}^{\prime(m)}$. Поэтому из 3) следует 2).

Из 2), учитывая 1), следует (3) и свойство $\sigma_{T}^{\prime(k)} \perp \sigma_{T}^{\prime(m)}(k \neq m)$.

Фиксируем $K \subseteq\{1, \ldots, n\},|K|=k$. Рассмотрим меру $\sigma_{K}=\sigma_{1} \times \cdots \times \sigma_{n}$, где $\sigma_{j}=\sigma_{T}^{\prime}$ при $j \neq \bar{K}$ и $\sigma_{j}=\delta$ иначе. Пусть $\nu_{K}(\cdot \mid c)$ - система канонических условных мер для меры $\sigma_{K}$ на слоях $C=\left\{\left(\lambda_{1}, \ldots, \lambda_{n}\right): \lambda_{1} \cdots \lambda_{n}=c\right\}\left(d \sigma_{K}=\nu_{K}(\cdot \mid c) d \sigma_{T}^{\prime(k)}\right)$. Ясно, что $\sigma_{T}^{\prime(k)}\left\{\left(\lambda_{1}, \ldots, \lambda_{n}\right): \lambda_{i}=\lambda_{j}\right\}=0(i \neq j)$. Поэтому, учитьвая (3), имеем для п.в. $c$ по мере $\sigma_{T}^{\prime}{ }^{(k)}$, что множество $\operatorname{supp} \nu_{K}(\cdot \mid c)$ состоит из $k$ ! точек, полученных из $\lambda(c)$ перестановками координат из $K$, где $\lambda(c)=\left(\lambda_{1}(c), \ldots, \lambda_{n}(c)\right), \lambda_{j}(c) \neq 1$ при $j \in K$ и $\lambda_{j}(c)=1$ иначе.

Учитывая, что $\sigma_{T}^{\prime(k)} \perp \sigma_{T}^{\prime(m)}(k \neq m)$, имеем

$$
\nu_{n}(\cdot \mid c) \sim \sum_{K:|K|=k} \nu_{K}(\cdot \mid c)
$$

для п.в. $c$ по мере $\sigma_{T}^{\prime}{ }^{(k)}$. Поэтому $\operatorname{supp} \nu_{n}(\cdot \mid c)$ содержит только точки, полученные из $\lambda(c)$ перестановкой $n$ координат для п.в. $c$ по мере $\sigma_{T}^{\prime}{ }^{(k)}$. Учитывая, что

$$
\sigma_{T}^{(n)} \sim \delta+\sum_{k=1}^{n} \sigma_{T}^{\prime(k)},
$$

получим, что верно 3). Лемма 1 доказана. 
ЗАмЕЧАНИЕ 4. Если свойство (*) верно при одном $n$, то $\sigma_{T}^{\prime}{ }^{(m)} \perp \sigma_{T}^{\prime(k)}(k \neq m$; $k, m \leqslant n)$. Действительно, $C_{\mathrm{sim}}(n)$ есть прямая сумма инвариантных подпространств $C_{i}$ $(i=0, n)$, где $\sigma_{T}^{\prime(i)}$ есть мера максимального спектрального типа оператора $T^{(n)}$ в $C_{i}$ $(i=1, n)$.

2. Для доказательства теоремы 1 нам понадобится следующий факт.

Лемма 2. Для любого $p \in \mathbb{Z}_{+}$

$$
T^{q_{n}+q_{n-p}} \rightarrow \frac{\left(3^{p+1}-1\right) E+2 \cdot\left(3^{p+1}+1\right) T^{-1}+\left(3^{p+1}-1\right) T^{-2}}{4 \cdot 3^{p+1}} \quad \text { npu } n \rightarrow \infty .
$$

ДокАЗАТЕльСтво. Прямое вычисление, вполне аналогичноепроверке, показьвает, что $T^{q_{n}} \rightarrow 0.5(E+T)$ при $n \rightarrow \infty$.

ДокАЗАТЕЛЬСТво ТЕОРемЫ 1 . Рассмотрим оператор $\tilde{T}^{(n)}$. Фиксируем типичньй по мере $\sigma_{T}^{\prime(n)}$ слой $C=\left\{\left(\lambda_{1}, \ldots, \lambda_{n}\right): \lambda_{1} \cdots \lambda_{n}=c\right\}$.

Докажем свойство 3 ) в лемме 1 . Обозначим

$$
P_{n}\left(\lambda_{1}, \ldots, \lambda_{n}, \theta\right)=\left(1+\theta \lambda_{1}+\lambda_{1}^{2}\right) \cdots\left(1+\theta \lambda_{n}+\lambda_{n}^{2}\right) .
$$

Если $T^{k_{i}} \rightarrow Q$, то $T^{(n)^{k_{i}}} \rightarrow Q^{(n)}$. Тогда для любого $f Q^{(n)} f \in C_{T^{(n)}}(f)$. Учитьвая лемму 2 , имеем

$$
P_{n}\left(\lambda_{1}, \ldots, \lambda_{n}, \theta_{p}\right) \in C_{\tilde{T}^{(n)}}(\mathbf{1})
$$

для разных $\theta_{p} \in \mathbb{R}$, т.е. $P_{n}\left(\lambda_{1}, \ldots, \lambda_{n}, \theta_{p}\right)$ являются константами на слое $C$ для п.в. $\left(\lambda_{1}\right.$, $\left.\ldots, \lambda_{n}\right)$ по мере $\nu_{n}(\cdot \mid c)$. Функция $P_{n}\left(\lambda_{1}, \ldots, \lambda_{n}, \theta\right)$ есть многочлен степени $n$ по переменной $\theta$ с постоянным старшим коэффициентом на слое $C$. Так как количество разных $\theta_{p} \geqslant n-1$, многочлен однозначно определен, т.е. его оставшиеся коэффициенты постоянны для п.в. $\left(\lambda_{1}, \ldots, \lambda_{n}\right)$ по мере $\nu_{n}(\cdot \mid c)$ на слое $C$. Поэтому он имеет один набор корней для п.в. $\left(\lambda_{1}, \ldots, \lambda_{n}\right)$, где $\lambda_{1} \cdots \lambda_{n}=c$. Учитывая равенство

$$
P_{n}\left(\lambda_{1}, \ldots, \lambda_{n}, \theta\right)=\lambda_{1} \cdots \lambda_{n}\left(\theta+\lambda_{1}+\bar{\lambda}_{1}\right) \cdots\left(\theta+\lambda_{n}+\bar{\lambda}_{n}\right) \quad\left(\left|\lambda_{1}\right| \equiv 1\right),
$$

имеем систему $\lambda_{i}+\bar{\lambda}_{i}=c_{i}$ для п.в. $\left(\lambda_{1}, \ldots, \lambda_{n}\right)$ на слое $C$ с точностью до перестановки набора $\left(c_{1}, \ldots, c_{n}\right)$. Ясно, что $c_{i} \in \mathbb{R}$, поэтому $\lambda_{i} \in\left\{\lambda_{i}^{(0)}, \bar{\lambda}_{i}^{(0)}\right\}$ для п.в. $\left(\lambda_{1}, \ldots, \lambda_{n}\right)$ на слое $C$ с точностью до перестановки набора $\left(\lambda_{1}^{(0)}, \ldots, \lambda_{n}^{(0)}\right)$. Поэтому $\operatorname{supp} \nu_{n}(\cdot \mid c)$ лежит в конечном множестве точек, полученных перестановкой координат из

$$
A=\left\{\left(\lambda_{1}, \ldots, \lambda_{n}\right): \lambda_{i} \in\left\{\lambda_{i}^{(0)}, \bar{\lambda}_{i}^{(0)}\right\}, i=1, \ldots, n\right\} .
$$

Так как $\nu_{n}(\cdot \mid c)$ симметрична, достаточно доказать, что $\nu_{n}(\cdot \mid c)(a)>0$ только для одного элемента $a$ из $A$.

Предположим, что $\nu_{n}(\cdot \mid c)\left(a_{i}\right)>0$, где $a_{i} \in A\left(i=1,2 ; a_{1} \neq a_{2}\right)$. Так как $a_{i} \in C$, то

$$
a_{i} \in\left\{\left(\lambda_{1}, \ldots, \lambda_{n}\right): \lambda_{j_{1}} \cdots \lambda_{j_{k}}= \pm 1\right\}=B_{ \pm}\left(j_{1}, \ldots, j_{k}\right)
$$

для некоторого набора $1 \leqslant j_{1}<j_{2}<\cdots<j_{k} \leqslant n$, где $k \geqslant 2$.

Так как борелевская мера $\sigma_{T}$ имеет атом только в точке 1 , отсюда нетрудно вывести, что

$$
\sigma_{T}^{n}\left(B_{ \pm}\left(j_{1}, \ldots, j_{k}\right) \backslash\{(1,1, \ldots, 1)\}\right)=0 .
$$

Поэтому на типичном слое $C$ имеем равенство

$$
\nu_{n}(\cdot \mid c)\left(B_{ \pm}\left(j_{1}, \ldots, j_{k}\right) \cap C \backslash\{(1,1, \ldots, 1)\}\right)=0 .
$$

Получим противоречие с предположением. Теорема 1 доказана. 
3. Классическим обобщением преобразования Чекана является преобразование $T$ Рудольфа-дель Юнко [7]. Здесь каждая башня $C_{n+1}$ получена разрезанием $C_{n}$ на $2^{n+1}$ подбашни и надставкой $(i+1)$-й подбашни наверх $i$-й, сделав вставку из 1 этажа между $2^{n}$-й и $\left(2^{n}+1\right)$-й подбашнями. В этом случае свойство $(*)$ было доказано в работе [5], так как $T^{k_{i}} \rightarrow \theta E+(1-\theta) T$ для любого $\theta \in[0,1]$.

4. Другим естественным обобщением преобразования Чекона является следующая лестничная конструкция. Фиксируем $r \in\{3,4, \ldots\}$. Каждая башня $C_{n+1}$ получена разрезанием $C_{n}$ на $r$ подбашен и надставкой $(i+1)$-й подбашни наверх $i$-й, сделав вставку из $i-1$ этажа между ними.

ТЕОРема 2. Для любого лестничного $T$ и любого $n M\left(T^{(n)}\right)=\{n, n(n-1), \ldots, n !\}$ $\theta$ const $\}^{\perp}$.

\section{СПИСОК ЦИТИРОВАННОЙ ЛИТЕРАТУРЫ}

[1] Junco A. del, Rahe M., Swanson L. Chacon's automorphism has minimal self-joinings // J. Anal. Math. 1980. V. 37. P. 276-284.

[2] Park K. K., Robinson E. A., Jr. The joinings within a class of $\mathbb{Z}^{2}$-actions // J. Anal. Math. 1991. V. 57. P. 1-36.

[3] Hamachi T., Silva C.E. On nonsingular Chacon transformations // Illinois J. Math. (to appear).

[4] Prihod'ko A. A., Ryzhikov V.V. Disjointness of the convolutions for Chacon's transformations // Colloq. Math. (to appear).

[5] Ageev O. N. On ergodic transformations with homogeneous spectrum // J. Dynamical Cont. Systems. 1999. V. 5. № 1. P. 149-152.

[6] Katok A. B. Constructions in ergodic theory // Unpublished Lecture Notes. 1987. V. 7. P. 229-248.

[7] Junco A. del, Rudolph D. J. A rank one, rigid, simple, prime map // Ergodic Theory Dynamical Systems. 1987. V. 7. P. 229-248.

[8] Корнфельд И. П., Синай Я. Г., Фомин С. В. Эргодическая теория. М.: Наука, 1980.

Московский государственный технический университет им. Н. Э. Баумана

Поступило

E-mail : ageev@mx.bmstu.ru

31.01 .2000 